\title{
Cosmological model with non-minimally coupled fermionic field
}

\author{
M. O. Ribas ${ }^{1,2}$, F. P. Devecchi ${ }^{1}$ and G. M. Kremer ${ }^{1}$ \\ ${ }^{1}$ Departamento de Física, Universidade Federal do Paraná, Curitiba, Brazil \\ ${ }^{2}$ Faculdades Integradas Espírita, Curitiba, Brazil
}

\begin{abstract}
A model for the Universe is proposed whose constituents are: (a) a dark energy field modeled by a fermionic field non-minimally coupled with the gravitational field, (b) a matter field which consists of pressureless baryonic and dark matter fields and (c) a field which represents the radiation and the neutrinos. The coupled system of Dirac's equations and Einstein field equations is solved numerically by considering a spatially flat homogeneous and isotropic Universe. It is shown that the proposed model can reproduce the expected red-shift behaviors of the deceleration parameter, of the density parameters of each constituent and of the luminosity distance. Furthermore, for small values of the red-shift the constant which couples the fermionic and gravitational fields has a remarkable influence on the density and deceleration parameters.
\end{abstract}

\section{Introduction}

In the last decade several works appeared in the literature with the aim the explanation of the astronomical observations [1] that the Universe is presently expanding with a positive acceleration. The great number of the works admits the existence of a component of the Universe - the so-called dark energy - which is the responsible for the present acceleration of the Universe, but whose nature still remains unknown. The most natural candidate for dark energy was initially a cosmological constant (see e.g. [2]), but other models for dark energy were also proposed. The most common models, also called quintessence models, make use of scalar fields and barotropic equations of state where the ratio between the pressure and energy density assumes negative values (e.g. [3] and the references therein). Other models consider that the dark energy field is described by exotic equations of state like the Chaplygin fluid [4] or the van der Waals fluid [5].

Some cosmological models were also investigated in the literature by considering fermions as sources of the gravitational field [6]. Recently, the authors [7] proposed a cosmological model in a dissipative Universe and showed that the fermions could be the responsible for accelerated regimes in the early Universe by simulating an inflaton field and in the present epoch by simulating a dark energy field. Another paper on this subject was published very recently [8].

In most of the works which investigate the role of the scalar field as source of the gravitational field it is common to consider it to be minimally coupled to the gravitational field. However, there exist some works in the literature [9] where it was investigated the effects of non-minimally coupled scalar fields during the evolution of the Universe.

The aim of the present work is to describe a spatially flat, homogeneous and isotropic Universe characterized by the Robertson-Walker metric and whose constituents are a dark energy field, a matter field and a field which represents the radiation and the neutrinos - from now on called radiation field. The dark energy field is modeled by a fermionic field which is non-minimally coupled 
with the gravitational field whereas the matter field represents the pressureless baryonic and dark matter fields. The radiation and matter fields are supposed to behave as non-interacting fields. The basic field equations for the evolution of the Universe follow from Dirac's equations and Einstein's field equations which are solved numerically for given initial conditions. The red-shift behaviors of the deceleration parameter, of the density parameters of each constituent and of the luminosity distance, which result from the numerical solutions, are compared with the available data set in order to verify the viability of the proposed model.

The manuscript is structured as follows: in Sec. II the Dirac equations and Einstein field equations are derived from the action of a non-minimally coupled fermionic field. The field equations for a flat, homogeneous and isotropic Universe are given in Sec. III, which are rewritten in terms of the red-shift in Sec. IV. In Sec. V the cosmological solutions are obtained by solving numerically the system of field equations for given initial conditions. We close the work with Sec. VI where we address to some remarks and sum up the results obtained. The metric signature used is $(+,-,-,-)$ and units have been chosen so that $8 \pi G=c=\hbar=1$.

\section{Non-minimally coupled fermionic field}

We are interested in investigate a Universe modeled by a mixture whose constituents are the fields of dark energy, matter and radiation. As was pointed out in the previous section, the dark energy is described by a fermionic field which is non-minimally coupled with the gravitational field, whereas the fields of matter and radiation are considered as non-interacting. The action for this model reads

$$
S=\int \sqrt{-g} d^{4} x\left[\frac{1}{2}(1-\xi \bar{\psi} \psi) R+\mathcal{L}_{f}+\mathcal{L}_{m}+\mathcal{L}_{r}\right],
$$

where $\mathcal{L}_{m}$ and $\mathcal{L}_{r}$ denote the Lagrangian densities of matter and radiation, respectively. Furthermore, $\psi$ and $\bar{\psi}$ are the spinor field and its adjoint, respectively, and $\xi$ is the coupling constant between the spinor field and the curvature scalar $R$. The spinor fields are treated here as classically commuting fields $\left[7,10\right.$. Finally, $\mathcal{L}_{f}$ is the Lagrangian density of the fermionic field which for massless fermions reads

$$
\mathcal{L}_{f}=\frac{\imath}{2}\left[\bar{\psi} \Gamma^{\mu} D_{\mu} \psi-\left(D_{\mu} \bar{\psi}\right) \Gamma^{\mu} \psi\right]-V .
$$

In eq. (2) $V$ - which is only a function of $\psi$ and $\bar{\psi}$ - describes the potential density of self-interaction between fermions. Moreover, $\Gamma^{\mu}=e_{a}^{\mu} \gamma^{a}$ are the generalized Dirac-Pauli matrices, $e_{\mu}^{a}$ denotes the tetrad or "vierbein" while the covariant derivatives are given by

$$
D_{\mu} \psi=\partial_{\mu} \psi-\Omega_{\mu} \psi, \quad D_{\mu} \bar{\psi}=\partial_{\mu} \bar{\psi}+\bar{\psi} \Omega_{\mu} .
$$

Above, the spin connection $\Omega_{\mu}$ is defined by

$$
\Omega_{\mu}=-\frac{1}{4} g_{\rho \sigma}\left[\Gamma_{\mu \delta}^{\rho}-e_{b}^{\rho} \partial_{\mu} e_{\delta}^{b}\right] \Gamma^{\sigma} \Gamma^{\delta},
$$

with $\Gamma_{\sigma \lambda}^{\nu}$ denoting the Christoffel symbols.

From Euler-Lagrange equations applied to the total Lagrangian density in eq. (1) it follows the Dirac equations for the spinor field and its adjoint coupled to the gravitational field, namely

$$
\begin{aligned}
& \imath \Gamma^{\mu} D_{\mu} \psi-\frac{d V}{d \bar{\psi}}-\frac{\xi}{2} R \psi=0, \\
& \imath D_{\mu} \bar{\psi} \Gamma^{\mu}+\frac{d V}{d \psi}+\frac{\xi}{2} R \bar{\psi}=0 .
\end{aligned}
$$


The variation of the action (11) with respect to the tetrad leads to Einstein's field equations

$$
R_{\mu \nu}-\frac{1}{2} g_{\mu \nu} R=-\frac{T_{\mu \nu}}{1-\xi \bar{\psi} \psi},
$$

where $T_{\mu \nu}$ is the total energy-momentum tensor of the sources which is a sum of the energymomentum tensor of the fields of fermions $T_{f}^{\mu \nu}$, matter $T_{m}^{\mu \nu}$ and radiation $T_{r}^{\mu \nu}$, i.e., $T^{\mu \nu}=T_{f}^{\mu \nu}+$ $T_{m}^{\mu \nu}+T_{r}^{\mu \nu}$

The symmetric form of the energy-momentum tensor of the fermions which follows from the variation of the action with respect to the tetrad reads

$$
\begin{gathered}
T_{f}^{\mu \nu}=\frac{\imath}{2}\left[\bar{\psi} \Gamma^{(\mu} D^{\nu)} \psi-D^{(\mu} \bar{\psi} \Gamma^{\nu)} \psi\right]-g^{\mu \nu} \mathcal{L}_{f} \\
-\xi\left(\mathcal{T}^{\mu \nu}-\mathcal{T}^{\sigma}{ }_{\sigma} g^{\mu \nu}\right) .
\end{gathered}
$$

Above, the parentheses around the indices denote the symmetric part of a tensor and it was introduced the tensor $\mathcal{T}_{\mu \nu}$ defined by

$$
\begin{gathered}
\mathcal{T}_{\mu \nu}=\left[D_{(\mu} D_{\nu)} \bar{\psi}\right] \psi+\bar{\psi}\left[D_{(\mu} D_{\nu)} \psi\right] \\
+2 D_{(\mu} \bar{\psi} D_{\nu)} \psi-\Gamma_{\mu \nu}^{\sigma}\left(D_{\sigma} \bar{\psi}\right) \psi-\Gamma_{\mu \nu}^{\sigma} \bar{\psi}\left(D_{\sigma} \psi\right) .
\end{gathered}
$$

\section{$3 \quad$ Field equations for Robertson-Walker metric}

From now on we shall investigate cosmological solutions which can be obtained from the above proposed model when applied to a homogeneous, isotropic and spatially flat Universe described by the Robertson-Walker metric $d s^{2}=d t^{2}-a(t)^{2} \delta_{i j} d x^{i} d x^{j}$, where $a(t)$ denotes the cosmic scale factor.

For the Robertson-Walker metric the components of the tetrad, spin connection and Dirac-Pauli matrices reduce to

$$
\begin{gathered}
e_{0}^{\mu}=\delta_{0}^{\mu}, \quad e_{i}^{\mu}=\frac{1}{a(t)} \delta_{i}^{\mu}, \quad \Omega_{0}=0, \quad \Omega_{i}=\frac{1}{2} \dot{a}(t) \gamma^{i} \gamma^{0} \\
\Gamma^{0}=\gamma^{0}, \quad \Gamma^{i}=\frac{1}{a(t)} \gamma^{i}, \quad \Gamma^{5}=-\imath \sqrt{-g} \Gamma^{0} \Gamma^{1} \Gamma^{2} \Gamma^{3}=\gamma^{5}
\end{gathered}
$$

Moreover, in this case the spinor field is an exclusive function of time and the Dirac equations (5) and (6) become

$$
\begin{aligned}
& \dot{\psi}+\frac{3}{2} H \psi+\imath \gamma^{0} \frac{d V}{d \bar{\psi}}+\imath \frac{\xi}{2} R \gamma^{0} \psi=0 \\
& \dot{\bar{\psi}}+\frac{3}{2} H \bar{\psi}-\imath \frac{d V}{d \psi} \gamma^{0}-\imath \frac{\xi}{2} R \bar{\psi} \gamma^{0}=0
\end{aligned}
$$

where $H=\dot{a}(t) / a(t)$ denotes the Hubble parameter and the dot represents a differentiation with respect to time.

For a homogeneous and isotropic Universe the total energy-momentum tensor of the sources of the gravitational field is written as $\left(T_{\nu}^{\mu}\right)=\operatorname{diag}(\rho,-p,-p,-p)$, where $\rho$ and $p$ denote the total energy density and pressure of the sources, respectively. In terms of the energy densities and pressures of the constituents, the total energy and pressure are written as

$$
\rho=\rho_{f}+\rho_{m}+\rho_{r}, \quad p=p_{f}+p_{m}+p_{r},
$$

i.e., as a sum of the energy densities and pressures of the fermionic, matter and radiation fields, respectively. 
From Einstein's field equations (77) it follows the modified forms of Friedmann and acceleration equations

$$
H^{2}=\frac{\rho}{3(1-\xi \bar{\psi} \psi)}, \quad \frac{\ddot{a}}{a}=-\frac{\rho+3 p}{6(1-\xi \bar{\psi} \psi)},
$$

respectively.

By differentiating the Friedmann equation $(15)_{1}$ with respect to time and taking into account: (i) the acceleration equation (15) 2 , (ii) the Dirac equations (12) and (13) and (iii) that the selfinteraction potential $V$ between the fermions does depend only on the bilinear $\Psi=\bar{\psi} \psi$, it follows the evolution equation for the total energy density of the sources of the gravitational field, namely

$$
\dot{\rho}+3 H(\rho+p)=-\frac{\rho \xi \dot{\Psi}}{(1-\xi \Psi)} .
$$

The above equation follows also from the Bianchi identities applied to the Einstein field equation (77). Note that due to the coupling between the fermionic and the gravitational fields, the right-hand side of (16) is not zero and represents the energy transfer between them. As it is well known, only two among the three equations given in (15) and (16) are linearly independent.

By assuming that the energy-momentum tensors of the constituents have the same form as the one for the total energy-momentum tensor, it follows from (8) and (9) that the energy density and pressure of the fermionic field read

$$
\begin{gathered}
\rho_{f}=V+3 \xi H \dot{\Psi}, \\
p_{f}=\frac{d V}{d \psi} \frac{\psi}{2}+\frac{\bar{\psi}}{2} \frac{d V}{d \bar{\psi}}-V+\frac{\xi}{2} R \Psi-\xi(\ddot{\Psi}+2 H \dot{\Psi}) .
\end{gathered}
$$

Note that the existence of a coupling between the fermionic and the gravitational fields leads to a dependence of the energy density and pressure of the fermionic field with respect to the energy density and pressure of all constituents through the Hubble parameter $H$ and the scalar curvature $R$.

After the matter and radiation decoupling both fields behave as non-interacting fields so that the evolution equations for their energy densities read

$$
\dot{\rho}_{m}+3 H \rho_{m}=0, \quad \dot{\rho}_{r}+4 H \rho_{r}=0,
$$

by taking into account that matter is considered as a pressureless fluid, i.e., $p_{m}=0$ whereas the radiation obeys the barotropic equation of state $p_{r}=\rho_{r} / 3$.

The evolution equation for the energy density of the fermionic field follows from (14), (16) and (19), yielding

$$
\dot{\rho}_{f}+3 H\left(\rho_{f}+p_{f}\right)=-\frac{\rho \xi \dot{\Psi}}{(1-\xi \Psi)},
$$

where the right-hand side of the above equation represents the energy transfer of the fermionic field to the gravitational field due to their coupling.

\section{Field Equations in terms of the red-shift}

In order to get cosmological solutions for the proposed model, the red-shift will be used as a variable instead of time thanks to the following relationships

$$
z=\frac{1}{a}-1, \quad \frac{d}{d t}=-H(1+z) \frac{d}{d z} .
$$


As a first step we note that that eqs. (19) can be easily integrated leading to the well-known dependence of the energy densities of the matter and radiation fields with the red-shift

$$
\rho_{m}(z)=\rho_{m}(0)(1+z)^{3}, \quad \rho_{r}(z)=\rho_{r}(0)(1+z)^{4},
$$

where $\rho_{m}(0)$ and $\rho_{r}(0)$ are the values of the energy densities at present time $z=0$.

Afterwards, the energy density and the pressure of the fermionic field are expressed in terms of the red-shift, yielding

$$
\begin{gathered}
\rho_{f}=V-3 \xi H^{2}(1+z) \Psi^{\prime}, \\
p_{f}=\left[\frac{2+\xi \Psi}{2(1-\xi \Psi)}\right]^{-1}\left\{\frac{d V}{d \Psi} \Psi-V-\xi\left[(1+z)^{2} H^{2} \Psi^{\prime \prime}\right.\right. \\
\left.\left.-(1+z) H^{2} \Psi^{\prime}+(1+z)^{2} H H^{\prime} \Psi^{\prime}\right]+\frac{\xi \Psi\left(\rho_{m}+\rho_{f}\right)}{2(1-\xi \Psi)}\right\},
\end{gathered}
$$

where the prime denotes the differentiation with respect to the red-shift $z$ and again by assuming that the self-interaction potential $V$ between the fermions does depend only on the bilinear $\Psi=\bar{\psi} \psi$. For a non-coupled fermionic field its energy density and pressure reduce to

$$
\rho_{f}=V, \quad p_{f}=\frac{d V}{d \Psi} \Psi-V .
$$

Finally, a system of two coupled differential equations for the bilinear $\Psi$ and for the Hubble parameter $H$ is obtained from the Dirac equations (12), (13) and from the Friedmann and acceleration equations (15), that reads

$$
\begin{gathered}
(1+z) H \Psi^{\prime \prime}-\left[2 H-(1+z) H^{\prime}\right] \Psi^{\prime}-3 H^{\prime} \Psi=0, \\
2 H(1+z)(1-\xi \Psi) H^{\prime}=\rho+p .
\end{gathered}
$$

For the determination of the cosmological solutions from the above equations we have also to specify how the self-interaction potential between the fermions $V$ depend on the bilinear $\Psi$. Here we suppose that it is given by the power law $V=\kappa /[\Psi(z)]^{\alpha}$, where $\kappa$ and $\alpha$ are two free parameters.

\section{Cosmological solutions}

For the search of numerical solutions of the system of differential equations (26) and (27) together with (22) through (24) we have to specify initial conditions for $\Psi(z), \Psi^{\prime}(z)$ and $H(z)$. Here we shall suppose that in the radiation dominated era at $z_{0}=10^{4}$ (say) the value of the bilinear is very small $\Psi\left(z_{0}\right)=10^{-5}$ with a smaller value for its derivative with respect to the red-shift $\Psi^{\prime}\left(z_{0}\right)=10^{-13}$ so that the fermionic field rolls very smoothly during the evolution of the Universe. Moreover, we shall adopt the following values for the density parameters $\Omega_{i}(z)=\rho_{i}(z) / \rho(z)$ at present time: $\Omega_{f}(0)=0.72, \Omega_{m}(0)=0.279916$ and $\Omega_{r}(0)=8.4 \times 10^{-5}$ (see e.g. [11). Hence the initial value for the dimensionless Hubble parameter can be obtained from Friedmann equation $(15)_{1}$ together with (22) and (23), yielding

$$
H\left(z_{0}\right)=\sqrt{\frac{\Omega_{m}(0)(1+z)^{3}+\Omega_{r}(0)(1+z)^{4}+\bar{\kappa} /\left[\Psi\left(z_{0}\right)\right]^{\alpha}}{3\left(1-\xi \Psi\left(z_{0}\right)\right)+3 \xi\left(1+z_{0}\right) \Psi^{\prime}\left(z_{0}\right)}},
$$

where $\bar{\kappa}$ is also a dimensionless quantity.

To find numerical solutions of the system of differential equations (26) and (27), still remains to fix values for the free parameters: (a) $\bar{\kappa}$ and $\alpha$ of the self-interaction potential between the fermions 


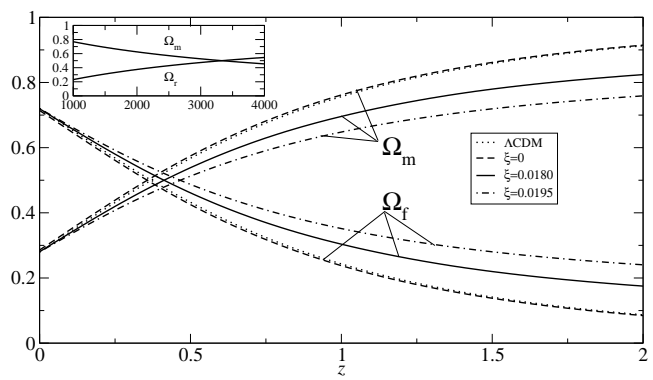

Figure 1: Density parameters as functions of the red-shift for the $\Lambda \mathrm{CDM}$ model and for the fermionic model with different values of $\xi$. Large frame $0 \leq z \leq 2$, small frame $1000 \leq z \leq 4000$.

and (b) the coupling constant between the fermionic field and the gravitational field $\xi$. The following values $\bar{\kappa}=0.6$ and $\alpha=1 / 10$ were found by imposing that the present value of the density parameter of the fermionic field matches the expected value $\Omega_{f}(0)=0.72$ when there is no coupling, i.e., when $\xi=0$. The search for values of these parameters by changing the initial conditions is exhaustible, specially due to the fact that the system of differential equations (26) and (27) is very unstable. Here we shall adopt the above quoted values.

In figure 1 the density parameters are plotted as functions of the red-shift in the range between $0 \leq z \leq 2$ (large frame) and in the range between $1000 \leq z \leq 4000$ (small frame). We infer from the large frame of this figure that the coupling parameter $\xi$ has influence on the decay of the dark energy field and the corresponding increase of the matter field. Moreover, the density parameter of the field of radiation in this range is very small. When $\xi=0$ there is no sensible difference between the $\Lambda \mathrm{CDM}$ model and the model where the fermions represent the dark energy field and there is no coupling. We note that by increasing the coupling parameter $\xi$ the decrease of the fermionic field and the corresponding increase of the matter field are less accentuated. This fact can be understood by noting that according to eq. (20) a part of the energy density of the fermionic field is transferred to the gravitational field due to its coupling. Furthermore, one can observe that the system of differential equations is very sensible to small changes of the coupling parameter. The density parameter of the fermionic field becomes negligible when $z \approx 5$ for the case when there is no coupling $(\xi=0)$ and when $z \approx 200$ for the other two cases $\xi=0.0180$ and $\xi=0.0195$, with a more accentuated decay of the former with respect to the latter. The density parameters of the radiation and matter fields are represented in the small frame in figure 1 , which shows that the equality between the matter and radiation occur at $z \approx 3300$. It is noteworthy that here we have taken into account the degrees of freedom of the relativistic neutrinos.

The deceleration parameter $q=1 / 2+3 p / 2 \rho$ is plotted in figure 2 as function of the red-shift. One can infer that the coupling parameter $\xi$ has also a very remarkable influence on the deceleration parameter $q$. It is important to note that the $\Lambda \mathrm{CDM}$ model furnishes a different solution with respect to the uncoupled fermionic model $(\xi=0)$. Indeed, although the values of $q$ coincide at large values of the red-shift $(z>1.5)$ their present values at $z=0$ and red-shifts $z_{t}$ that correspond to the transition from a decelerated to an accelerated regime read: (a) $\Lambda$ CDM model $q(0)=-0.58$ and $z_{t}=0.73$ and (b) uncoupled model $q(0)=-0.68$ and $z_{t}=0.80$. The corresponding values for $\xi \neq 0$ are: (c) $q(0)=-0.58$ and $z_{t}=0.64$ for $\xi=0.0180$ and (d) $q(0)=-0.49$ and $z_{t}=0.50$ for $\xi=0.0195$. Apart the last case all others fit more or less the experimental values for the deceleration parameter, namely, $q(0)=-0.74 \pm 0.18$ (see [12]) and $z_{t}=0.46 \pm 0.13$ (see [13]).

In figure 3 it is plotted the difference between the apparent $m$ and the absolute magnitude $M$ of 


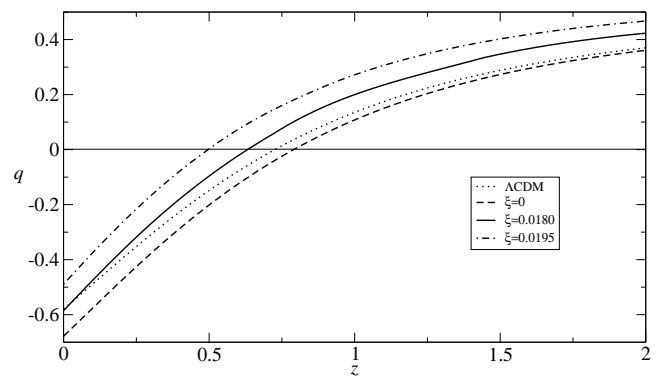

Figure 2: Deceleration parameters as functions of the red-shift for the $\Lambda$ CDM model and for the fermionic model with different values of $\xi$.

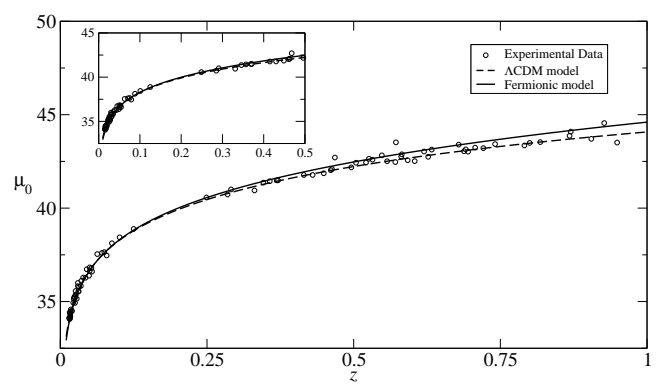

Figure 3: $\mu_{0}$ as function of the red-shift $z$ for the $\Lambda$ CDM model and for the fermionic model. Large frame $0 \leq z \leq 1$, small frame $0 \leq z \leq 0.5$.

a source, denoted by $\mu_{0}$ and defined by

$$
\mu_{0}=m-M=5 \log \left\{(1+z) \int_{0}^{z} \frac{d z^{\prime}}{H\left(z^{\prime}\right)}\right\}+25,
$$

where the quantity between braces represents the luminosity distance in Mpc. The circles in this figure correspond to the experimental data for super-novae of type Ia taken from the recent work by Riess et al. 14. It is important to call attention to the fact that the values of the coupling constant $\xi$ chosen above do not lead to sensible differences in the values of $\mu_{0}$ so that only one curve for the fermionic model is represented in the figure. One can infer from the small frame of figure 3 that there is a very good agreement of the curves of the $\Lambda \mathrm{CDM}$ model and of the fermionic model with the experimental data for red-shifts in the range $0 \leq z \leq 0.5$. From the large frame of this figure one observes that there exist some differences between the curves of the two models at higher red-shifts $0.5 \leq z \leq 1$.

\section{$6 \quad$ Final remarks and conclusions}

In this work we have considered a classical fermionic field non-minimally coupled with the gravitational field. Classical spinors were discussed by Armendáriz-Picón and Greene in Ref. [6] and for more details one is referred to this work. Here we point out that: (i) the expectation value of a spinorial field in a physical state is a complex number and not a Grassmannian number and (ii) the spinorial field can be treated classically if its state is close to the vacuum. 
Recently, exact cosmological solutions for a Universe described by a Robertson-Walker metric and modeled by a neutral scalar field and neutrinos were obtained in the work [15. The model of this work is different from the model presented here since in the former the neutrinos are considered as a Majorana fermion field while the neutral scalar field represents the acceleron of the mass-varying neutrinos.

One important point which deserves attention is the one concerning the equivalence of the above description with the one in which the Einstein's field equations is written as

$$
R_{\mu \nu}-\frac{1}{2} g_{\mu \nu} R=-\tilde{T}_{\mu \nu}
$$

where $\tilde{T}_{\mu \nu}=\tilde{T}_{f}^{\mu \nu}+T_{m}^{\mu \nu}+T_{r}^{\mu \nu}$, with

$$
\begin{aligned}
& \tilde{T}_{f}^{\mu \nu}=\frac{\imath}{2}\left[\bar{\psi} \Gamma^{(\mu} D^{\nu)} \psi-D^{(\mu} \bar{\psi} \Gamma^{\nu)} \psi\right]-g^{\mu \nu} \mathcal{L}_{f} \\
& -\xi\left(\mathcal{T}^{\mu \nu}-\mathcal{T}^{\sigma}{ }_{\sigma} g^{\mu \nu}\right)-\xi \bar{\psi} \psi\left(R_{\mu \nu}-\frac{1}{2} g_{\mu \nu} R\right) .
\end{aligned}
$$

In this case we have $T^{\tilde{\mu} \nu}{ }_{; \nu}=0$ thanks to the Bianchi identities. If we write the total energymomentum tensor as $\left(\tilde{T}_{\nu}^{\mu}\right)=\operatorname{diag}(\tilde{\rho},-\tilde{p},-\tilde{p},-\tilde{p})-$ so that $\tilde{\rho}$ and $\tilde{p}$ represent the total energy density and the total pressure, respectively - it follows

$$
H^{2}=\frac{\tilde{\rho}}{3}, \quad \frac{\ddot{a}}{a}=-\frac{\tilde{\rho}+3 \tilde{p}}{6}, \quad \dot{\tilde{\rho}}+3 H(\tilde{\rho}+\tilde{p})=0,
$$

which are the usual form of the Friedmann, acceleration and energy conservation equations, respectively.

Equations (32) are not in contradiction with the corresponding eqs. (15) and (16), since one can prove that the energy density and pressure of the latter case are related with those of former case by

$$
\tilde{\rho}=\frac{\rho}{(1-\xi \Psi)}, \quad \tilde{p}=\frac{p}{(1-\xi \Psi)} .
$$

Hence, it is straightforward to obtain eqs. (15) and (16) from eqs. (32) and (33) by the use of Dirac's equations (12) and (13).

Another remark is that the non-minimally coupling is important only at small red-shifts and its effect becomes negligible at high red-shifts where the matter-radiation decouples and the nucleosynthesis begins. Moreover, the method adopted here was to solve the system of differential equations (26) and (27) by specifying small values for $\Psi\left(z_{0}\right)$ and $\Psi^{\prime}\left(z_{0}\right)$ at a high red-shift $z_{0}$ so that the fermionic field rolls very smoothly during the evolution of the Universe. Another method follows by combining equations (12) and (13) so that the resulting equation leads to

$$
\frac{\Psi(z)}{\Psi\left(z_{0}\right)}=\frac{(1+z)^{3}}{\left(1+z_{0}\right)^{3}}
$$

From the above equation one can obtain that the energy density of the fermionic field reads

$$
\rho_{f}=\left[\frac{K}{[\Psi(z)]^{n}}-\frac{3 \xi\left[\rho_{m}(z)+\rho_{r}(z)\right] \Psi(z)}{[1-\xi \Psi(z)]}\right]\left[\frac{1-\xi \Psi(z)}{1+2 \xi \Psi(z)}\right] .
$$

Now by specifying an initial condition to the bilinear $\Psi(z)$ one can made a similar analysis on the behavior of the density parameters, of the deceleration parameter and of the luminosity distance as was done in Sec. V. 
As a last remark we call attention to the fact that we have considered a very simple type self-interaction potential which depends only on the bilinear $\bar{\psi} \psi$. With this choice, the fermionic field seems to behave as a bosonic field, but a comparison of the field equations and solutions of the present work with those obtained by Binder and Kremer in Ref. 9] for a bosonic field nonminimally coupled with the gravitational field shows that the solutions are quite different. A more general self-interaction potential should also depend on the pseudo-scalar invariant $\left(i \bar{\psi} \gamma^{5} \psi\right)^{2}$ as the one investigated by the authors in Ref. [7. This work is in progress and shall appear in a forthcoming paper.

To sum up: (a) the proposed model is very sensible concerning the choice of the initial conditions and the choice of the free parameters $\xi, \bar{\kappa}$ and $\alpha$; (b) the density parameter of the fermionic and matter fields decrease more slowly with respect to the red-shift when the fermions are coupled $(\xi \neq 0)$ due to a more efficient energy transfer between the fermionic and the gravitational fields; (c) by increasing the coupling constant $\xi$ the transition from a decelerated to an accelerated regime occurs at earlier red-shifts and (d) there is no significant difference in parameter $\mu_{0}$-which is related to the luminosity distance - for coupled and uncoupled fermionic fields.

\section{Acknowledgments}

The authors FPD and GMK acknowledge the support by Conselho Nacional de Desenvolvimento Científico e Tecnológico (CNPq).

\section{References}

[1] RIESS A. G. et al. Astron. J. 1161998 1009;Perlmutter S. et al. Astrophys. J. 5171999565.

[2] CARROLL S. M. Liv. Rev. Rel. 420011.

[3] PEEBLES P. J. E. and RATRA B. Rev. Mod. Phys. 752003 559; SZYDEOWSKI M., KUREK A. and KRAWIECK A. Phys. Lett. B 6422006171.

[4] CAPOzZIEllo S., DE MARTino S. and FAlAngA M. Phys. Lett. A 2992002 494; CAPOZZIELlO S., CARDONE V. F., CARLONI S., DE MARTINO S., FAlANGA M., TROISI A. and BRUNI M. J. Cosmol. Astropart. Phys. 042005 005; KREMER G. M. Phys. Rev. D 682003 123507; KREMER G. M. Gen. Relativ. Gravit. 362004 1423; KREMER G. M. Gen. Relativ. Gravit. 392007965.

[5] KAMENSHCHIK A. YU., MOSCHELlA U. and PASQUIER V. Phys. Lett. B 5112001 265; FABRIS J. C., GONÇALVES S. V. B. and DE SOUZA P. E. Gen. Relativ. Gravit. 342002 53; BENTO M. C., BERTOlAMi O. and SEN A. A. Phys. Rev. D 662002 043507; KREMER G. M. Gen. Relativ. Gravit. 3520031459.

[6] OBUKHOV Y. N. Phys. Lett. A 1821993 214; SAHA B. and SHIKIN G. N. Gen. Relativ. Gravit. 291997 1099; SAHA B. Phys. Rev. D 642001 123501; ARMENDÁRIZ-PICÓN C. and GREENE P. B. Gen. Relativ. Gravit. 352003 1637; SAHA B. and BOYADJIEV T. Phys. Rev. D 692004 124010; SAHA B. Phys. Rev. D 692004124006.

[7] RIBAS M. O., DEVECCHI F. P. and KREMER G. M. Phys. Rev. D 722005123502.

[8] SAHA B. Phys. Rev. D 742006124030. 
[9] MADSEn M. S. Class. Quant. Grav. 51988 627; UZAN J. P. Phys. Rev. D 591999 123510; CHIBA T. Phys. Rev. D 601999 083508; AMENDOLA L. Phys. Rev. D 601999 043501; ESPOSITO-FARĖSE G. and POLARSKI D. Phys. Rev. D 632001 063504; FARAONI V. Int. J. Theor. Phys. 402001 2259; ABRAMO L.R., BRENIG L. and GUNZIG E. Phys. Lett. B 5492002 13; BINDER J. B. and KREMER G. M. Braz. J. Phys. 352005 1038; BINDER J. B. and KREMER G. M. Gen. Relativ. Gravit. 382006857.

[10] RYDER L. H. Quantum Field Theory (Cambridge University Press, Cambridge, 1996).

[11] FUKUGitA M. and PEEBLES P. J. E. Astrophys. J. 6162004643.

[12] VIREY J.-M. et al. Phys. Rev. D 722005061302.

[13] RIESS A. G. et al. Astrophys. J. 6072004665.

[14] RIESS A. G. et al. Astrophys. J. 659200798.

[15] BALANTEKin A. B. and DERELI T. Phys. Rev. D 752007024039. 